\title{
BMJ Open Non-absorbable versus absorbable sutures for anterior colporrhaphy: study protocol for a randomised controlled trial in South Korea
}

\author{
Myung Jae Jeon (D) , ${ }^{1}$ Dong Hoon Suh, ${ }^{2}$ Chul Hong Kim, ${ }^{3}$ Hyun-Hee Cho, ${ }^{4}$ \\ Jung-Ho Shin, ${ }^{5}$ Sa Ra Lee, ${ }^{6}$ Yong Wook Jung, ${ }^{7}$ Soo Rim Kim, ${ }^{8}$ Mi Kyung Kong ${ }^{9}$
}

To cite: Jeon MJ, Suh DH, $\mathrm{Kim} \mathrm{CH}$, et al. Non-absorbable versus absorbable sutures for anterior colporrhaphy: study protocol for a randomised controlled trial in South Korea. BMJ Open 2020;10:e034218. doi:10.1136/ bmjopen-2019-034218

- Prepublication history and additional material for this paper are available online. To view these files, please visit the journal online (http://dx.doi org/10.1136/bmjopen-2019034218).

Received 10 September 2019 Revised 25 April 2020 Accepted 19 May 2020

Check for updates

(C) Author(s) (or their employer(s)) 2020. Re-use permitted under CC BY-NC. No commercial re-use. See rights and permissions. Published by BMJ.

For numbered affiliations see end of article.

Correspondence to Professor Myung Jae Jeon; jeonmj@snu.ac.kr

\section{ABSTRACT}

Introduction The anterior vaginal wall is the segment most commonly affected by prolapse. Traditionally, anterior vaginal wall prolapse is repaired via anterior colporrhaphy, which is known to have a high recurrence rate. Several factors might affect the outcome of anterior colporrhaphy, and the use of absorbable sutures might also be associated with the high recurrence rate because the sutures might not be able to retain adequate strength until the plicated pubocervical fascia remodels and regains maximum tensile strength. Nonetheless, no comparative data exist about the relative efficacy and safety of anterior colporrhaphy using non-absorbable versus absorbable sutures. The objective of this study is to compare the surgical outcomes of anterior colporrhaphy using nonabsorbable sutures with those of anterior colporrhaphy using absorbable sutures.

Methods and analysis This is a randomised, multicentre, superiority trial. Anterior colporrhaphy will be performed in a traditional manner with midline plication of the fibromuscular layer using either non-absorbable or absorbable sutures. The primary outcome is composite surgical success 1 year after surgery defined as the absence of all of the following: (1) anterior vaginal descent beyond the hymen, (2) the presence of vaginal bulge symptoms and (3) retreatment for recurrent anterior vaginal wall prolapse with either surgery or pessary. The secondary outcomes include the individual components of the composite primary end point, anatomical outcomes, condition-specific quality of life and adverse events related to anterior colporrhaphy. The planned number of participants is 192.

Ethics and dissemination This study was approved by the Institutional Review Board of Seoul National University Hospital (H-1810-037-977). The results of the study will be published in peer-reviewed journals, and the findings will be presented at scientific meetings.

Trial registration number NCT03736811

\section{INTRODUCTION}

The anterior vaginal wall is the segment most commonly affected by prolapse. ${ }^{1}$ Traditionally, anterior vaginal wall prolapse is repaired with anterior colporrhaphy, which is known to have a high recurrence rate. When strict
Strengths and limitations of this study

- This is the first study to compare surgical outcomes of anterior colporrhaphy using non-absorbable sutures with those of anterior colporrhaphy using absorbable sutures.

- The findings will be strengthened by the multicentre, randomised design, masking both participants and outcome assessors to randomisation assignment, and the use of validated measures to assess anatomic and symptomatic outcomes.

- Our study participants consist of Korean women, and therefore, the findings may not apply to more racially diverse population.

anatomic criteria are used (pelvic organ prolapse quantification (POPQ) stage $\geq 2$ ), recurrence rates after anterior colporrhaphy are reported up to $70 \% .^{2}$ The use of mesh repair may decrease the recurrence rate, but the complication rate is higher compared with anterior colporrhaphy. ${ }^{3}$ Therefore, anterior colporrhaphy remains the most common procedure performed to correct anterior vaginal wall prolapse.

Anterior colporrhaphy involves the midline plication of fibromuscular layers (pubocervical fascia) of the anterior vaginal wall. Absorbable sutures are most often used. ${ }^{4}$ Several factors, including the preoperative degree of pelvic organ prolapse, might affect the outcome of anterior colporrhaphy, ${ }^{56}$ and the use of absorbable sutures might also be associated with the high recurrence rate after anterior colporrhaphy. Wound healing is divided into three phases: inflammation (1-4 days), proliferation (5-20 days) and remodelling (21 days to 2 years). ${ }^{7}$ The wound begins to regain its tensile strength from the proliferative phase (up to $15 \%-20 \%$ of its original tissue strength) and gain maximum strength in the remodelling phase (approximately 
$70 \%-80 \%$ of its original tissue strength).$^{8}$ Meanwhile, absorbable sutures such as polyglactin 910 or polydioxanone commonly used for anterior colporrhaphy lose at least half of their tensile strength within 1 month and the entire strength within 2-3 months. ${ }^{8}$ This may be sufficient for wounds under little or no tension. However, plicated pubocervical fascia may need more time to remodel and regain maximum tensile strength because the increased abdominal pressure generated from daily activity, coughing, straining, exercise and so forth may act as a factor that impedes wound healing. ${ }^{9}$

Data from several clinical trials support this theory. First, most cases of recurrence after anterior colporrhaphy are detected within the first postoperative year. ${ }^{2} 10$ Second, the short-term follow-up study of uterosacral ligament suspension for apical vaginal prolapse has reported that the use of non-absorbable sutures is associated with a lower failure rate than the use of absorbable sutures, even though no failure has been detected until 6 months after surgery. ${ }^{11}$ Nonetheless, no comparative data exist about the relative efficacy and safety of anterior colporrhaphy using non-absorbable versus absorbable sutures. The objective of this study is to compare the surgical outcomes of anterior colporrhaphy using non-absorbable sutures with those of anterior colporrhaphy using absorbable sutures.

\section{METHODS AND ANALYSIS \\ Study design}

This trial is a multicentre, prospective, randomised trial conducted with the aim of determining the superiority of anterior colporrhaphy using non-absorbable sutures over absorbable sutures with regard to the primary outcome. The study will be a single-blind study, as it is impossible to blind the study surgeon for the surgical procedure to which the subject is assigned. However, all outcome assessors and the subjects will be blinded to the treatment assignment. Postoperative follow-up will take place after 5 weeks, 6 months and 12 months. Patients will undergo a standard gynaecological examination that includes the POPQ and will fill in questionnaires. The design is presented in figure 1 . This study protocol was approved on 8 November 2018 and this manuscript details the protocol on the latest version (V.1.5) approved on 02 April 2020, which adheres to the Standard Protocol Items: Recommendations for Clinical Interventional Trials guidelines (online supplementary file 1 ).

\section{Study setting}

This study will be conducted in nine tertiary hospitals in South Korea. Enrolment, treatment and data collection will be standardised by all sites according to the approved study protocol and this manuscript.

\section{Participants and recruitment}

The study population will consist of women aged 30 years or older who have symptomatic anterior vaginal

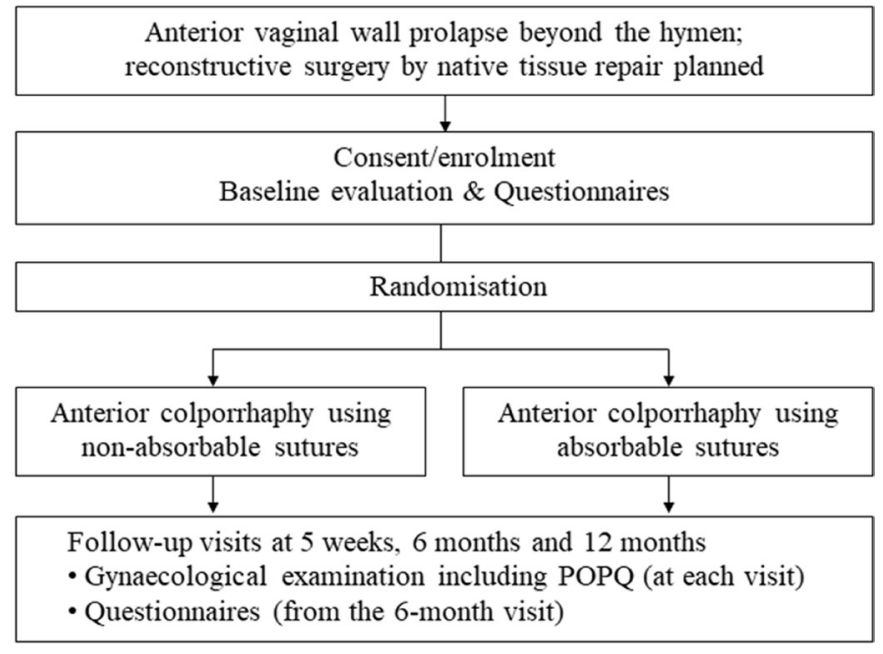

Figure 1 Study flow chart. POPQ, pelvic organ prolapse quantification.

wall prolapse and have opted for reconstructive surgery with native tissue repair. After screening for eligibility, information regarding the study will be provided and written informed consent will be obtained by the research staff (online supplementary file 2). The inclusion and exclusion criteria are presented in box 1 . Recruitment commenced on 8 November 2018.

\section{Randomisation}

Randomisation will be performed through a website using a computer-generated randomisation table in the operating room by the study surgeon. The subjects will be assigned in a 1:1 ratio to receive anterior colporrhaphy using either non-absorbable sutures or absorbable sutures. The randomisation will be stratified according to the preoperative stage of anterior vaginal wall prolapse, and all subjects will receive a unique study number.

To prevent unmasking the actual procedures, the medical records will indicate the actual surgical procedure without stating the suture materials used for anterior colporrhaphy. Intraoperative data collection will be conducted by the study surgeon rather than by other research staff.

\section{Intervention}

Participants will undergo reconstructive surgery for prolapse, including anterior colporrhaphy using the assigned suture materials under general or spinal anaesthesia. Anterior colporrhaphy will be performed in a traditional manner with midline plication of the fibromuscular layer using either non-absorbable (polyester (Ethibond Excel) or polypropylene (Prolene); Ethicon, Somerville, New Jersey, USA) or absorbable (polyglactin 910 (Vicryl) or polydioxanone (PDS II); Ethicon) sutures. ${ }^{4}$

Concomitant procedures will be performed as intended prior to surgery. Women with a uterus in situ will undergo hysterectomy, and all women will receive an apical suspension procedure, including uterosacral ligament 


\section{Box 1 Inclusion and exclusion criteria}

Inclusion criteria

- Anterior vaginal wall prolapse beyond the hymen (POPQ point $\mathrm{Ba}$ $>0$ ).

- Vaginal bulge symptoms as indicated by an affirmative response to the following question from the PFDI-20: Do you usually have a bulge or something falling out that you can see or feel in your vaginal area?

Reconstructive surgery via native tissue repair is planned.

Exclusion criteria

- Recurrent anterior vaginal wall prolapse.

- Reconstructive surgery using mesh or obliterative surgery is planned.

Known pelvic malignancy.

- Current systemic glucocorticoid or immunosuppressant treatment.

- Subject wishes to retain her uterus.

Subject is unable or unwilling to participate.

PFDI, Pelvic Floor Distress Inventory; POPQ, pelvic organ prolapse quantification

suspension, sacrospinous ligament fixation and iliococcygeal suspension with both delayed absorbable and permanent sutures, according to the preference of the surgeon because the loss of apical support is usually present when anterior vaginal wall prolapse extends beyond the hymen, and surgical correction of the anterior wall may fail unless the apex is adequately supported. ${ }^{12}{ }^{13}$ Posterior colporrhaphy and incontinence surgery will also be performed, if indicated (ie, women with $\mathrm{Bp} \geq-1$ or documented urodynamic stress incontinence). Participating surgeons will be required to have performed a minimum of 20 of each procedure prior to beginning subject enrolment to eliminate a learning curve effect.

All women will receive perioperative antibiotics. Postoperatively a vaginal pack will be placed and removed within 24 hours. A voiding trial will take place on postoperative day 2. A postvoid residual volume of $150 \mathrm{~mL}$ or greater will be considered abnormal. Patients with elevated postvoid residual volumes will continue mechanical bladder drainage either via a continuous transurethral Foley catheter or intermittent self-catheterisation until the postvoid residual volumes are consistently less than $150 \mathrm{~mL}$. Patients will receive analgesics in accordance with the local hospital protocol. All patients will be advised to abstain from heavy physical work for a minimal period of 6 weeks.

\section{Data collection}

At baseline, the following data will be collected: demographics and medical history data (age, body mass index, parity, menopausal and hormone therapy status, current smoking, previous hysterectomy and previous anti-incontinence surgery, and medical comorbidities (diabetes mellitus, connective tissue disorders)), and data from the standardised POPQ examination in a $45^{\circ}$ upright sitting position during maximal Valsalva. ${ }^{14}$ Patients will be asked to complete the validated questionnaires regarding condition-specific quality of life (the Korean version of
Pelvic Floor Distress Inventory short form questionnaire (PFDI-20)). ${ }^{15}$

Scheduled in-person follow-ups will occur at 5 weeks, 6 months and 12 months. Each check-up will include a clinical examination including POPQ and written questionnaires identical to those at baseline (from the 6-month visit). In addition, an update of current medications, an assessment of new or continuing pelvic floor disorders and adverse events that occurred since the previous evaluation will be obtained by the study coordinator at each visit. All data will be anonymised and collected using case report forms by examiners or trained research coordinators at each participating centre who are blinded to the treatment assignment. Quality checks will be performed by all centres and reviewed every 3 months by an independent data monitoring committee.

\section{Primary and secondary outcome measures}

Initially the primary outcome was surgical success assessed at 1 and 5 years after surgery. As our sample size calculation could not reflect the 5-year outcome, we changed to assess the primary outcome only for 1 year after surgery during the revision of this manuscript. Surgical success is defined as the absence of all of the following: (1) anterior vaginal descent beyond the hymen, (2) presence of vaginal bulge symptoms and (3) retreatment for recurrent anterior vaginal wall prolapse by either surgery or pessary. The secondary outcomes include the individual components of the composite primary end point, anatomical outcomes (the rate of suboptimal anatomical outcome in each compartment (POPQ point $\mathrm{Ba}, \mathrm{C}$ or $\mathrm{Bp} \geq-1$ ), change in POPQ values from baseline (POPQ Ba, C, Bp and total vaginal length)), condition-specific quality of life (change in PFDI-20 scores from baseline), and the rate of adverse events related to anterior colporrhaphy that occurred since baseline (both intraoperative (bladder injury, ureteral obstruction, massive bleeding] and postoperative (haematoma, vesico-vaginal fistula, ureteral obstruction, urinary tract infection, incomplete bladder emptying, overactive bladder or stress incontinence symptoms, suture erosion, vaginal wound dehiscence, infection or granulation tissue, etc)).

\section{Sample size and power considerations}

The statistical power calculation is based on a comparison of a binary primary outcome. Previous studies using similar definitions to ours demonstrated that the 1-year surgical success rates after anterior colporrhaphy were $97 \%$ with non-absorbable sutures and $85 \%$ with absorbable sutures. ${ }^{11} 16$ Based on this information, we estimate that at least 86 patients are needed in each treatment group for $80 \%$ power to detect a $12 \%$ difference in the primary outcome measure, with a two-tailed type I error of $5 \%$, at 1 year after surgery. Considering a $10 \%$ drop-out rate, we will recruit and randomise 192 subjects in this protocol. 


\section{Data analysis}

The baseline characteristics between the two groups will be compared using a two sample t-test or Mann-Whitney $\mathrm{U}$ test for continuous variables and a $\chi^{2}$ test or Fisher's exact test for categorical variables.

The analyses for all outcome measures will be performed on both an intention-to-treat and as-treated basis, but the principal analysis will be the intention-to-treat analysis for the effectiveness (all outcomes except adverse events) and the as-treated analysis for the safety (adverse events).

The primary outcome will be analysed using a $\chi^{2}$ test based on the intention-to-treat principle after missing values for it are imputed with multiple imputation. In addition, as a sensitivity analysis, the group difference in the primary outcome will be compared using a generalised linear mixed-effect model (mixed-effects logistic regression) with group as a fixed effect and hospital as a random effect to account for correlation of results within each hospital. If the baseline variables are found to be significantly different between groups, they will be included as covariates in the mixed-effects model.

Secondary outcomes will be analysed as follows: The individual components of the composite primary end point and the rate of suboptimal anatomical outcomes in each compartment will be analysed similar to the primary outcome. Changes in POPQ values and PFDI-20 scores will be analysed using the linear mixed models including hospital as a random effect, with adjustment for baselines. The rate of adverse events related to anterior colporrhaphy will be compared with a $\chi^{2}$ test or Fisher's exact test

\section{Patient and public involvement}

Neither the patients nor the public were involved in the design, conduct, reporting or dissemination of this study.

\section{Data monitoring}

Data monitoring will be performed every 3 months by an independent data monitoring committee. The committee will monitor protocol deviations, violations, data quality and serious adverse events. No interim analysis is planned during this trial.

\section{Ethics approval and dissemination}

The study will be conducted in accordance with the principles of the Declaration of Helsinki and 'good clinical practice' guidelines. This trial was approved by the Institutional Review Board (SNUH 1810-037-977). Prior to randomisation informed consent will be obtained. All participantidentifiable data, such as consent forms, screening and identification logs, will be stored in the investigator site files, accessible only to delegated members of the study team. Any personal information will be neither recorded in case report forms nor shared with others. The datasets used and/or analysed after completing the study will be available from the corresponding author on reasonable requests. The results of the study will be published in peer-reviewed journals, and the findings will be presented at scientific meetings. Authorship will be determined by the guidelines set out by the International Committee of Medical Journal Editors.

\section{Author affiliations}

${ }^{1}$ Obstetrics and Gynecology, Seoul National University College of Medicine, Seoul, The Republic of Korea

${ }^{2}$ Obstetrics and Gynecology, Seoul National University Bundang Hospital, Seongnam, The Republic of Korea

${ }^{3}$ Obstetrics and Gynecology, Chonnam National University Medical School, Gwangju, The Republic of Korea

${ }^{4}$ Obstetrics and Gynecology, The Catholic University of Korea, Eunpyeong St. Mary's Hospital, Seoul, The Republic of Korea

${ }^{5}$ Obstetrics and Gynecology, Korea University College of Medicine, Seoul, The Republic of Korea

${ }^{6}$ Obstetrics and Gynecology, University of Ulsan College of Medicine, Asan Medical Center, Seoul, The Republic of Korea

${ }^{7}$ Obstetrics and Gynecology, CHA Gangnam Medical Center, CHA University College of Medicine, Seoul, The Republic of Korea

${ }^{8}$ Obstetrics and Gynecology, International St. Mary's Hospital, Catholic Kwandong University College of Medicine, Incheon, The Republic of Korea

${ }^{9}$ Obstetrics and Gynecology, Institute of Women's Life Medical Science, Yonsei University College of Medicine, Seoul, The Republic of Korea

Contributors All authors contributed to the conception and design of the research protocol. MJJ is the principal investigator of the whole study. DHS, CHK, HHC, JHS, SRL, YWJ, SRK and MKK are the site principal investigators of each research centre. MJJ drafted the original manuscript, and DHS, CHK, HHC, JHS, SRL, YWJ, SRK and MKK revised it. All authors approved the final version.

Funding The authors have not declared a specific grant for this research from any funding agency in the public, commercial or not-for-profit sectors.

Competing interests None declared.

Patient consent for publication Not required.

Provenance and peer review Not commissioned; externally peer reviewed.

Open access This is an open access article distributed in accordance with the Creative Commons Attribution Non Commercial (CC BY-NC 4.0) license, which permits others to distribute, remix, adapt, build upon this work non-commercially, and license their derivative works on different terms, provided the original work is properly cited, appropriate credit is given, any changes made indicated, and the use is non-commercial. See: http://creativecommons.org/licenses/by-nc/4.0/.

ORCID iD

Myung Jae Jeon http://orcid.org/0000-0001-5582-1488

\section{REFERENCES}

1 Hendrix SL, Clark A, Nygaard I, et al. Pelvic organ prolapse in the women's health Initiative: gravity and gravidity. Am J Obstet Gynecol 2002;186:1160-6.

2 Weber AM, Walters MD, Piedmonte MR, et al. Anterior colporrhaphy: a randomized trial of three surgical techniques. Am J Obstet Gynecol 2001;185:1299-306.

3 Maher C, Feiner B, Baessler K, et al. Surgery for women with anterior compartment prolapse. Cochrane Database Syst Rev 2016;11:CD004014.

4 Halpern-Elenskaia K, Umek W, Bodner-Adler B, et al. Anterior colporrhaphy: a standard operation? systematic review of the technical aspects of a common procedure in randomized controlled trials. Int Urogynecol J 2018;29:781-8.

5 Jeon MJ, Chung SM, Jung HJ, et al. Risk factors for the recurrence of pelvic organ prolapse. Gynecol Obstet Invest 2008;66:268-73.

6 Margulies RU, Rogers MAM, Morgan DM. Outcomes of transvaginal uterosacral ligament suspension: systematic review and metaanalysis. Am J Obstet Gynecol 2010;202:124-34.

7 Maxson S, Lopez EA, Yoo D, et al. Concise review: role of mesenchymal stem cells in wound repair. Stem Cells Transl Med 2012;1:142-9.

8 Greenberg JA, Clark RM. Advances in suture material for obstetric and gynecologic surgery. Rev Obstet Gynecol 2009;2:146-58. 
9 Murphy M. Restrictions and limitations after pelvic floor surgery: what's the evidence? Curr Opin Obstet Gynecol 2017;29:349-53.

10 Dietz HP, Hankins KJ, Wong V. The natural history of cystocele recurrence. Int Urogynecol J 2014;25:1053-7.

11 Chung CP, Miskimins R, Kuehl TJ, et al. Permanent suture used in uterosacral ligament suspension offers better anatomical support than delayed absorbable suture. Int Urogynecol J 2012;23:223-7.

12 Rooney K, Kenton K, Mueller ER, et al. Advanced anterior vaginal wall prolapse is highly correlated with apical prolapse. Am J Obstet Gynecol 2006;195:1837-40.

13 Eilber KS, Alperin M, Khan A, et al. Outcomes of vaginal prolapse surgery among female Medicare beneficiaries: the role of apical support. Obstet Gynecol 2013;122:981-7.
14 Bump RC, Mattiasson A, Bø K, et al. The standardization of terminology of female pelvic organ prolapse and pelvic floor dysfunction. Am J Obstet Gynecol 1996;175:10-17.

15 Yoo E-H, Jeon MJ, Ahn K-H, et al. Translation and linguistic validation of Korean version of short form of pelvic floor distress inventory-20, pelvic floor impact questionnaire-7. Obstet Gynecol Sci 2013;56:330-2.

16 Barber MD, Brubaker L, Burgio KL, et al. Comparison of 2 transvaginal surgical approaches and perioperative behavioral therapy for apical vaginal prolapse: the optimal randomized trial. JAMA 2014;311:1023-34. 